\title{
Kulinarische Transfers aus anderen Regionen und Kulturräumen: der Niederschlag in westdeutschen und österreichischen Kochbüchern 1950-1980
}

\author{
EIN WERKSTATTBERICHT ÜBER EINEN „MIXED METHOdS“-ANSATZ AUS KULTURWIS- \\ Senschaftlichem Close Reading und digitalen Methoden
}

Christoph Bläsi \& Edith Blaschitz

Keywords: Cookbooks, Culinary Transfers, Mixed Methods

\begin{abstract}
This project investigates how culinary transfers of 'foreign' food are reflected in Austrian and German cookbooks from 1950 to 1980. For this, methods from Digital Humanities are combined with cultural studies approaches.
\end{abstract}

\begin{abstract}
1 Einleitung
Im dargestellten Forschungsprojekt wird untersucht, wie sich kulinarische Transfers aus anderen Regionen und Kulturräumen in westdeutschen und österreichischen Kochbüchern der Jahre 1950-1980 widerspiegeln. Erste Ergebnisse des interdisziplinären Projektes, in dem Daten sowohl mit Methoden der Digital Humanities als auch mit hermeneutisch-kulturwissenschaftlichen Herangehensweisen analysiert werden, liegen nun vor.
\end{abstract}

\section{Das Kochbuch als Medium und Forschungsgegenstand}

Obgleich das Kochbuch ein am Buchmarkt anhaltend relevantes Genre ist, das 2017 in Deutschland mit 25,6 \% den höchsten Anteil im Ratgebersegment einnahm¹, findet es als Untersuchungsgegenstand in der Buchwissenschaft unangemessen wenig Beachtung. Die Buchwissenschaft sieht sich hier grundsätzlich zumindest auch zuständig, insoweit sie zwar zunächst Bücher aus Produktions-, Distributions- und Rezeptionsperspektive, insbesondere im Hinblick auf Aspekte von Materialität und Medialität der Buchkommunikation, beforscht, sich als dann gewissermaßen Disziplin vernachlässigter Gegenstände aber nicht selten auch inhaltlicher Aspekte non-fiktionaler Buchgenres annimmt; ein Beispiel dafür zitieren wir unten.

In den kulturwissenschaftlich orientierten Medien- und den Literaturwissenschaften ${ }^{2}$ wird das vorwiegend non-fiktionale Kochbuch ebenso wenig wahrgenommen. ${ }^{3}$ Kochbücher werden hingegen in

1 Börsenverein des Deutschen Buchhandels, Buch und Buchhandel in Zahlen 2018. Zahlen, Fakten und Analysen zur wirtschaftlichen Entwicklung, Frankfurt am Main 2018, 18.

2 Jens Ruchatz, Kochbücher und andere Kochmedien, Vortrag 19.11.2018, Workshop „Kulturelle Aneignungs- und Bewahrungsprozesse und deren mediale Resonanzen am Beispiel von ,Kochwissen“', Johannes-Gutenberg-Universität Mainz.

3 Siehe etwa Hermann CöLFTEN, Vom Kochrezept zur Kochanleitung: sprachliche und mediale Aspekte einer verständlichen Vermittlung von Kochkenntnissen, in: Unikate: Berichte aus Forschung und Lehre 30 (2007), 84-93; Jonatan LeER u. a., 
den geisteswissenschaftlich orientierten Food Studies (Ethnologie, Geschichtswissenschaften etc.) herangezogen, wenn Entstehung und identitätsschaffender bzw. -wahrender Charakter von Regionaloder Nationalküchen untersucht werden ${ }^{4}$. Die Digital Humanities nehmen sich Kochbüchern an, fokussieren dabei im Wesentlichen aber die Produktion annotierter digitaler Kochbucheditionen. ${ }^{5}$

War das Kochbuch, das vor allem ab dem späten 19. Jahrhundert eine nennenswerte Verbreitung fand, zunächst ein vorwiegend der Kochpraxis dienendes Textbuch, das mit Rezepten Handlungsanleitungen zur Zubereitung von Gerichten bot, haben sich ab den 1920er-Jahren mehrere der genannten Bestimmungsgrößen sukzessive aufgeweicht: Ein modernes Kochbuch ist, so kann man z. B. mit Bateman et al. ${ }^{6}$ sagen, heute typischerweise ein komplexer multimodaler Text, bei dem mit sprachlichen Elementen (immer mehr mit narrativen Textpassagen, oft zur Kontextualisierung des Rezeptes), auch mit Bildern (aus der „Food“-, aber ebenso aus der so genannten „Mood“-Fotografie) sowie in hohem Maße mit den Möglichkeiten des Layouts (Spalten, Weißräume, Boxen etc.) gearbeitet wird. Zu den konstitutiven Bestandteilen auf der inhaltlichen Ebene gehören Rezepte, die im Kern aus Zutatenliste und Zubereitungsanweisungen bestehen, aber eben, wie erwähnt, auch Rahmentexte (z. B. zu Grundlagen des Kochens, zu Klassen von Gerichten, zum Kontext wie zu Regionen oder Kulturräumen etc.) sowie Bilder und ggf. andere Illustrationen.

Zusätzlich zu den genannten inhaltlichen und formalen Entwicklungen des Genres - und z.T. direkt mit diesem korrespondierend - fand auch eine Funktionserweiterung des Kochbuchs statt: Es wird nicht mehr notwendigerweise nur als Ratgeber für die Zubereitung von Gerichten verwendet, sondern kann z. B. auch als Ausdruck des eigenen Habitus gesammelt und präsentiert werden. Außerdem kann es als „Phantasiematerial“7 dienen, um sich in eine narrative Welt ,transportieren“ zu lassen, wie man das sonst nur als Nutzungsoption bei fiktionalen Texten kennt ${ }^{8}$.

\section{Regionale, nationale und ,fremde` Küche im Kochbuch}

Kochbücher sind immer von den jeweiligen gesellschaftlichen, politischen und sozioökonomischen Rahmenbedingungen beeinflusst. Mit einer massenhaften Verbreitung von Kochbüchern im 19. Jahrhundert erfolgte ein Vergesellschaftungsprozess von ,Kochwissen': Das Wissen über die Zubereitung von Speisen und das Repertoire an Gerichten wird nicht mehr nur individuell weitergegeben (also z. B. von der Mutter an die Tochter), sondern auch über das Medium Buch. Mit der Mediatisierung findet ein Normierungsprozess statt. Kochbücher sind somit nicht nur mediale Repräsentanten

Food and media: practices, distinctions and heterotopiaLondon, NY 2016.

4 Siehe exemplarisch: Kirsten Schlegel-Matthies, Regionale Speisen in deutschen Kochbüchern des 19. und 20. Jahrhunderts, in: Hans Jürgen Teuteberg u. a., Hg., Essen und kulturelle Identität. Europäische Perspektiven, Berlin 1997, 212-227; Nicola Humble, Culinary Pleasures. A History of Britain through Cookbooks, London 2005; Ronda L. BRULOTTE / Michael A. Dı Gıovine, Edible Identities: Food as Cultural Heritage, Farnham 2014; Maren MöHRING, Fremdes Essen. Die Geschichte der ausländischen Gastronomie in der Bundesrepublik Deutschland, München 2012; Regina FRISCH, Biografie eines Kochbuchs: das Bayerische Kochbuch erzählt Kulturgeschichte, Regensburg 2016; Peter NACCARATO u. a., Representing Italy through Food, London 2018.

5 Siehe exemplarisch Helmut W. KLUG / Karin KRANICH, Das Edieren von handschriftlichen Kochrezepttexten am Weg ins digitale Zeitalter - Zur Neuedition des Tegernseer Wirtschaftsbuches, in: Thomas Bein, Hg., Vom Nutzen der Editionen. Zur Bedeutung moderner Editorik für die Erforschung von Literatur- und Kulturgeschichte, Berlin / Boston 2015, $121-137$.

6 John Bateman / Janina Wildfeuer / Tuomo Hilppala, Multimodality. Foundations, Research and Analysis. A ProblemOriented Introduction, Berlin 2017.

7 Klaus-Ulrich PECH (1998), zit. in: Anke Vogel / David Oels, Wie und warum man Kochbücher verwendet. Eine empirische Studie, in: David Oels / Michael Schikowski, Hg., Non-Fiktion - Ratgeber, Hannover 2012, 127-142, hier 138.

8 Melanie C. Green u. a., Understanding Media Enjoyment: The Role of Transportation Into Narrative Worlds, in: Communication Theory 14/4 (2004), 311-327. 
gesellschaftlicher Prozesse, sondern sie konstruieren diese mit, schreiben sie im wahrsten Sinne des Wortes fest. So wurden im Prozess des „Nation Buildings“ im 19. Jahrhundert bestimmte Speisen zum Kanon einer Nationalküche in Kochbüchern zusammengefasst. Diese trägt zur Konstituierung einer eigenen nationalen Identität bei - und damit auch zur Abgrenzung nach außen (also der Konstruktion des ,Eigenen“ im Gegensatz zum ,Fremden').

\section{Untersuchung des Niederschlags kulinarischer Transfers aus anderen Regionen und Kulturräumen in westdeutschen und österreichischen Kochbücher 1950-1980}

Das Projekt der Donau-Universität Krems und der Johannes-Gutenberg-Universität Mainz untersucht den Niederschlag von kulinarischen Transfers aus Regionen und Kulturräumen, die sich im Untersuchungszeitraum nicht innerhalb des westdeutschen oder des österreichischen Staatsgebietes befanden, anhand eines ausgewählten Bestandes an deutschen und österreichischen Kochbüchern aus dem Zeitraum von 1950 bis 1980. Unterstützt wird das Projekt - vor allem wegen seines Digital Humanities-Anteils - von mainzed, dem Mainzer Zentrum für Digitalität in den Geistes- und Kulturwissenschaften.

Der gewählte Zeitraum 1950 bis 1980 wird in der Bundesrepublik Deutschland und in Österreich neben anderen Transformationen mit einem massiven ,Internationalisierungsschub“ verbunden. Bei der Untersuchung der - Vorkriegstraditionen geschuldeten - kontinuierlichen Verwendung bzw. Neuaufnahme von Zutaten, Zubereitungsweisen und ganzen Rezepten aus anderen Regionen und Kulturräumen müssen diese dafür nicht nur als solche identifiziert werden, sonders es stellen sich auch Fragen wie die, ob erkennbar wird, mit welchen Konnotationen, Bewertungen etc. durch die Autor*innen bzw. Herausgeber*innen diese Aufnahme erfolgt ist und ob sich nachvollziehbar Vermutungen anstellen lassen, welche Vorstellungen und Zielgruppenmodellierungen der Grund für die Aufnahme gewesen sein könnten.

Das hier darzustellende Forschungsprojekt betrachtet die Aufnahme von Zutaten, Zubereitungsweisen und Rezepten aus anderen Regionen und Kulturräumen in Kochbücher als eine Folge gesellschaftlicher Kontinuitäten als auch von Transformationen verschiedener Art. Kochbücher ,framen' dabei oft explizit, zumindest aber implizit, die Aufnahme von bestimmten Rezepten (aus welchen Gründen diese auch immer redaktionell erfolgt ist). Der Begriff des ,Fremden“ fasst - sozusagen als ein Begriff unserer ,Werkstattsprache - zwar immer wieder sehr plastisch, wie das, um das es in vielen Fällen geht, vortheoretisch gesehen werden kann - an Beispielen wie dem ungarischen Gulasch, das nach dem Ende der k.u.k.-Monarchie für Österreich zwar staatspolitisch entfremdet, für die österreichische Standardküche als Gericht aber weiterhin als ,Eigenes' betrachtet wurde, sieht man aber, dass das Gegensatzpaar aus ,Eigenem' und ,Fremdem' als zentrale Dichotomie zu viel theoretischen Ballast mitbringt und überdies auch nicht den ganzen Raum der uns interessierenden Phänomene aufspannt. Wir haben deshalb die Arbeit am Thema zwar mit einem Pilotprojekt mit den das ,Fremde' anspielenden Begriffen von Aneignung und Bewahrung im Titel (,Kulturelle Aneignungs- und Bewahrungsprozesse und deren mediale Resonanzen am Beispiel von ,Kochwissen“") gestartet ${ }^{9}$, sind im Verlauf der Forschungsarbeit aber zu der Einsicht gekommen, dass es uns präziser - und so fassen wir das ja auch in dieser Darstellung - um den Niederschlag von kulinarischen Transferprozessen in Kochbüchern geht.

9 Pilotprojektdauer: November 2017-November 2018, gefördert von: Inneruniversitäre Forschungsförderung (Stufe I), Johannes-Gutenberg-Universität Mainz. 


\subsection{Unser grundsätzliches Vorgehen}

Schon weil mehrere der oben angedeuteten bestehenden Forschungsstränge - vor allem natürlich der zur kulturellen Rolle und Funktion von Nahrungszubereitung und Essen und der zum Medium Kochbuch - für die gegebene Fragestellung Aufmerksamkeit verdienen, bietet sich im Kern eine Forschungszusammenarbeit aus einer Kulturwissenschaftlerin und einem Buchwissenschaftler, wegen des methodischen Ansatzes unterstützt von Computerphilologen, unmittelbar an. Die sich daraus ergebende grundsätzliche Arbeitsweise kann folgendermaßen beschrieben werden: Aufgrund der kulturwissenschaftlich-philologischen Betrachtung von Kochbüchern gewonnene Fragen und Arbeitshypothesen werden mit computerphilologischen Methoden überprüft, die Ergebnisse davon hermeneutisch - also vor allem mit Bezug auf relevante Kontexte - ausgewertet und aus diesen - in einem nächsten Durchgang des zirkulären Vorgehens - neue Fragen und Arbeitshypothesen entwickelt. Die Entscheidung für einen „Mixed Methods“-Ansatz beruht nicht zuletzt auf der Tatsache, dass z. B. der oben erwähnte Grund für die Aufnahme eines Rezeptes meist nicht oder nicht explizit im untersuchten Kochbuch selbst ablesbar ist, sondern dass Vermutungen im Hinblick auf den Aufnahmegrund einer Kontextualisierung mit anderen Texten und auch gesellschaftlichen etc. Gegebenheiten bedürfen.

\subsection{Wo wir stehen}

Als Grundlage für unsere Pilotarbeit haben wir zunächst zwei Standard-Kochbücher - das „Franz Ruhm Kochbuch“ und das „Dr. Oetker Schulkochbuch“10 von einem Dienstleister scannen, der optischen Zeichenerkennung unterziehen und dann nach dem TEI-Tite-Standard vorstrukturieren lassen. Parallel dazu haben wir eine größere Anzahl von Kochbüchern aus dem Betrachtungszeitraum untersucht und Arbeitshypothesen zunächst v.a. dazu entwickelt, wie sich kulinarische Transfers in Kochbüchern niederschlagen können bzw. welche textlichen (und bildlichen!) Gegebenheiten als Marker-Kandidaten für ,Transfer-Rezepte' herangezogen werden können. Zu den auf diese Weise identifizierten Markern gehören vor allem in spezifizierten Referenzkochbüchern (aus der Zeit vor 1950) noch nicht oder noch nicht in der Häufigkeit vorkommende Zutaten (z. B. Olivenöl), Bezeichnungen, in denen Namen von Orten aus dem Quellgebiet des Transfers vorkommen (z. B. „bosnische Suppe“) oder sonstige sprachliche Elemente aus den Sprachen des Quellgebietes (z. B. „à la mère“). Relevant sind aber auch Rahmentexte, die Transfers implizit oder explizit identifizieren oder sogar bewerten, indem sie z. B. auf Migrations- oder Urlaubserfahrungen anspielen.

Einfachere aus diesen kulturwissenschaftlich erarbeiteten Markern (z. B. Städte- und Ländernamen, Zutaten wie Käsesorten) haben wir dann in einem nächsten Schritt zur Grundlage genommen, um in den digitalisierten Kochbüchern automatisiert zu überprüfen, ob die Operationalisierung hermeneutisch plausible Ergebnisse bringt. Die Nutzung von autoritativen Listen von Städten und Ländern sowie von Lebensmittelgruppen, z. B. Käsesorten, hat hier in der Tat unmittelbar Ergebnisse gebracht - also Kandidaten für ,Transfer-Rezepte‘ identifiziert, einige von diesen durchaus unerwartet.

10 Franz Ruнm, Das Franz Ruhm Kochbuch. Das Standardwerk der Wiener und österreichischen Küche, Wien 1980; Dr. Oetker Schulkochbuch, Bielefeld 1952. 


\section{Was wir gelernt haben und was jetzt ansteht}

Wir haben gesehen, dass die ursprünglich als ein nächster Schritt vorgesehene Tiefenstrukturierung der Kochbücher (z. B. die Auszeichnung von Mengen und Zutaten) in TEI (lite oder ,proper') weniger prioritär sein dürfte als angenommen - viele der ins Auge gefassten computerphilologischen Methoden (z. B. für die erwähnten Bewertungen: Emotion Analysis) erfordern nicht zwingend strukturierte Texte. Auf der anderen Seite wurde sehr klar, dass die Beobachtung der Entwicklung über die gesamte Untersuchungszeit konstitutiv wichtig ist, da es eine Frequenzentwicklung von relativen Vorkommen von Namen und Begriffen gibt, die mit der Unterscheidung „Vorkommen / Nicht-Vorkommen“ nicht angemessen erfasst werden kann. Dafür ist die Vergrößerung des Korpus an digitalisierten Kochbüchern essentiell. Jenseits des Begriffs des ,Fremden', den wir als zu operationalisierendes Konstrukt hinter uns gelassen haben, müssen wir die Liste der Marker theoriebasiert erweitern und diese um weitere Operationalisierungen des Niederschlags kulinarischer Transfers aus anderen Regionen und Kulturräumen ergänzen. Auch scheint über den Einbezug von Referenzkochbüchern aus der Zeit vor 1950 eine Erweiterung um weiteres sprachliches ,Referenz-Material' wichtig; zu diesem gehören etwa Werke aus der Kochausbildung sowie Texte, die Erfahrungen und Bewertungen von Migration oder von Urlaub, aber z. B. auch von gesunder Lebensweise - ein weiterer möglicher Aufnahmegrund von Rezepten anderer Provenienz - erfassen. Neben der Verfeinerung des Instrumentariums zur Analyse von Textuellem gilt es im nächsten Schritt zumindest auch leichtgewichtigere Methoden der Bildanalyse einzubeziehen (wir sehen Kochbücher im Untersuchungszeitraum weiterhin als primär textlich konstituierte Artefakte), da sich ,Fremdheit', auch das ein Ergebnis der ersten Phase, in einigen Fällen erst über bildliches Material erschließt.

\section{Literaturverzeichnis}

John Bateman / Janina Wildfeuer / Tuomo HiIPpala, Multimodality. Foundations, Research and Analysis - A Problem-Oriented Introduction, Berlin 2017.

Börsenverein des Deutschen Buchhandels, Buch und Buchhandel in Zahlen 2018. Zahlen, Fakten und Analysen zur wirtschaftlichen Entwicklung, Frankfurt am Main 2018.

Ronda L. BrulotTe / Michael A. Dı Giovine, Edible Identities: Food as Cultural Heritage, Farnham 2014.

Hermann CÖLFTEN, Vom Kochrezept zur Kochanleitung: sprachliche und mediale Aspekte einer verständlichen Vermittlung von Kochkenntnissen, in: Unikate: Berichte aus Forschung und Lehre 30 (2007), 84-93.

Regina FRISCH, Biografie eines Kochbuchs: das Bayerische Kochbuch erzählt Kulturgeschichte, Regensburg 2016.

Melanie C. GreEN u. a., Understanding Media Enjoyment: The Role of Transportation Into Narrative Worlds, in: Communication Theory 14/4 (2004), 311-327.

Nicola Humble, Culinary Pleasures. A History of Britain through Cookbooks, London 2005.

Helmut W. KLUG / Karin KRANICH, Das Edieren von handschriftlichen Kochrezepttexten am Weg ins digitale Zeitalter - Zur Neuedition des Tegernseer Wirtschaftsbuches, in: Thomas Bein, Hg., Vom Nutzen der Editionen. Zur Bedeutung moderner Editorik für die Erforschung von Literatur- und Kulturgeschichte, Berlin / Boston 2015, 121-137.

Jonatan LeER u. a., Food and media: practices, distinctions and heterotopia, London / NY 2016.

Maren MöHRING, Fremdes Essen. Die Geschichte der ausländischen Gastronomie in der Bundesrepublik Deutschland, München 2012. 
Peter Naccarato u. a., Representing Italy through Food, London 2018.

Dr. Oetker Schulkochbuch, Bielefeld 1952.

Klaus-Ulrich PECH (1998), zit. in: Anke Vogel / David Oels, Wie und warum man Kochbücher verwendet. Eine empirische Studie, in: David Oels / Michael Schikowski, Hg., Non-Fiktion - Ratgeber, Hannover 2012, 127-142.

Jens Ruchatz, Kochbücher und andere Kochmedien, Vortrag 19.11.2018, Workshop „Kulturelle Aneignungs- und Bewahrungsprozesse und deren mediale Resonanzen am Beispiel von ,Kochwissen“, Johannes-Gutenberg-Universität Mainz.

Franz Ruнm, Das Franz Ruhm Kochbuch. Das Standardwerk der Wiener und österreichischen Küche, Wien 1980.

Kirsten Schlegel-MatTHies, Regionale Speisen in deutschen Kochbüchern des 19. und 20. Jahrhunderts, in: Hans Jürgen Teuteberg u. a., Hg., Essen und kulturelle Identität. Europäische Perspektiven, Berlin 1997, 212-227. 